\title{
What Drives Metal-Surface Step Bunching in Graphene Chemical Vapor Deposition?
}

\author{
Ding Yi, ${ }^{1}$ Da Luo, ${ }^{1}$ Zhu-Jun Wang, ${ }^{2}$ Jichen Dong, ${ }^{1}$ Xu Zhang, ${ }^{1}$ Marc-Georg Willinger, ${ }^{2, *}$ \\ Rodney S. Ruoff, ${ }^{1,3,4, \dagger}$ and Feng Ding ${ }^{1,3, \#}$ \\ ${ }^{1}$ Center for Multidimensional Carbon Materials (CMCM), Institute for Basic Science (IBS), Ulsan 44919, Republic of Korea \\ ${ }^{2}$ Department of Inorganic Chemistry, Fritz Haber Institute of the Max Planck Society, \\ Berlin-Dahlem D-14195, Germany \\ ${ }^{3}$ School of Materials Science and Engineering, Ulsan National Institute of Science and Technology (UNIST), \\ Ulsan 44919, Republic of Korea \\ ${ }^{4}$ Department of Chemistry, Ulsan National Institute of Science and Technology (UNIST), \\ Ulsan 44919, Republic of Korea
}

(Received 1 January 2018; published 12 June 2018)

\begin{abstract}
Compressive strain relaxation of a chemical vapor deposition (CVD) grown graphene overlayer has been considered to be the main driving force behind metal surface step bunching (SB) in CVD graphene growth. Here, by combining theoretical studies with experimental observations, we prove that the SB can occur even in the absence of a compressive strain, is enabled by the rapid diffusion of metal adatoms beneath the graphene and is driven by the release of the bending energy of the graphene overlayer in the vicinity of steps. Based on this new understanding, we explain a number of experimental observations such as the temperature dependence of SB, and how SB depends on the thickness of the graphene film. This study also shows that SB is a general phenomenon that can occur in all substrates covered by films of two-dimensional (2D) materials.
\end{abstract}

DOI: 10.1103/PhysRevLett.120.246101

The chemical vapor deposition (CVD) process for the synthesis of graphene has received intense attention, and a variety of transition metals, e.g., $\mathrm{Cu}, \mathrm{Ni}, \mathrm{Pt}, \mathrm{Ru}, \mathrm{Rh}$, and $\mathrm{Ir}$ [1-14] have been investigated as substrates for graphene CVD growth (among others). Among these, $\mathrm{Cu}$, which has the advantage of easy single layer graphene (SLG) growth via a self-limiting growth mechanism [1], is most promising for large area, high-quality graphene synthesis [15-21]. High-quality polycrystalline SLG films with areas in $\mathrm{m}^{2}$ and single crystalline SLG with domain areas of the order of several $\mathrm{cm}^{2}$ have been reported [22-24].

It is widely acknowledged that a smooth substrate is essential to obtain high-quality graphene [25-27]. Therefore, in CVD experiments, the $\mathrm{Cu}$ substrate is usually annealed at temperatures close to its melting point so as to achieve an ultraflat surface for graphene synthesis. But even when grown on an ultraflat $\mathrm{Cu}$ surface, after cooling down, it was often found that the as-grown graphene islands were always localized on the rough areas of the $\mathrm{Cu}$ surface containing multiple steps [28-33]. In contrast, areas with pure $\mathrm{Cu}$, not covered by graphene, were found to be mostly atomically flat [30-33]. Depending on the type of crystal facet, the experimental conditions of graphene CVD growth, and the thickness of the graphene layer, the step-heights of these "macro steps" range from 3 to $50 \mathrm{~nm}$, which is 15 to 250 times higher than the height of a single atomic step on the $\mathrm{Cu}(111)$ surface $(0.21 \mathrm{~nm})$ (see summary in the Supplemental Material [34]).
Previous works have proposed that macrosteps on a metal surface are formed by step bunching (SB), which occurs during the cooling stage of the CVD process and that $\mathrm{SB}$ is driven by the relaxation of compressive strain in the graphene overlayer. $\mathrm{Cu}$ has a relatively larger thermal expansion coefficient of $\sim 2 \times 10^{-5} \mathrm{~K}^{-1}$, while that of graphene is almost negligible (and also negative) [45-47]. Hence, when the graphene/Cu sample is cooled down from $\sim 1000^{\circ} \mathrm{C}$ to room temperature, a compressive strain of $\sim 2 \%$ will be exerted on the graphene layer [26,47-49]. It has been therefore proposed that the relaxation of this compressive strain is the driving force behind $\mathrm{SB}$ on the $\mathrm{Cu}$ surface directly underneath the graphene layer [31,50-52]. This assumption was supported by the experimental observation that $\mathrm{Cu}$ surfaces without graphene coverage are atomically flat [30-33].

In Figs. 1(a)-1(c), we present in situ observation of the SB process on a graphene-covered $\mathrm{Cu}$ surface during cooling [34]. Figure 1(b) shows that only the areas covered with graphene show the presence of macrosteps and Fig. 1(c) shows that these steps disappear once the graphene overlayer is etched away. This observation confirms that graphene coverage is essential for SB to occur. Our in situ observation further shows that there are certain regions on the $\mathrm{Cu}$ surface that even when covered by graphene [marked with $A, B, C$, and $D$ in Fig. 1(a)], remain atomically flat. This is due to the fact that low index surfaces (e.g., 111 or 001 surfaces) have no steps. Based on the above observations, we conclude that both graphene coverage and the prior existence of steps are essential conditions for SB and a model of the SB process is 

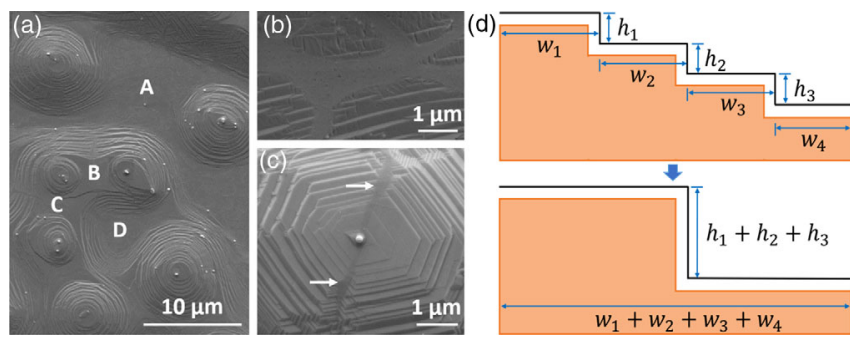

FIG. 1. (a)-(c) In situ scanning electron microscopy images of graphene-covered $\mathrm{Cu}$ surfaces taken at $\sim 600^{\circ} \mathrm{C}$ during cooling following graphene growth. $A, B, C$, and $D$ in (a) mark four areas covered with graphene, but where no step bunches appear. (d) Schematic illustrations of a model to describe metal surface SB beneath graphene.

presented in Fig. 1(d). Here it is interesting to note that as shown in the model, the total length of the graphene layer on the $\mathrm{Cu}$ surface before and after SB are exactly the same and, consequently, SB cannot release any compressive strain in the graphene layer. Thus, the previously proposed explanation of the mechanism of metal surface SB beneath a graphene layer needs to be reviewed.

To test the above argument that step bunching does not serve to release compressive strain on the graphene layer, a $\mathrm{Cu}(111)$ foil was covered with graphene flakes transferred at room temperature, and this $\mathrm{Cu}(111)$ foil was heated at different temperatures and then cooled back to room temperature [34]. The Raman spectrum of the transferred graphene on the $\mathrm{Cu}$ foil shows a $G$ peak at $1581 \mathrm{~cm}^{-1}$ indicating that the transferred graphene flake is stress-free [Fig. 2(f)] [53]. Thus, when a $\mathrm{Cu}$ foil is heated to a high temperature, the graphene flake formed has either zero strain if it slides freely on the $\mathrm{Cu}$ surface or has a tensile strain (as the $\mathrm{Cu}$ has significantly elongated) if it does not slide freely. In either case, there is no compressive strain on the graphene flake. If the annealing temperature is increased, macrosteps gradually become visible, starting from when $T>300^{\circ} \mathrm{C}$ (Fig. 2). At the temperature of $\sim 600-700^{\circ} \mathrm{C}$, step heights reach $20-40 \mathrm{~nm}$, which is very close to those observed in graphene CVD growth. Raman spectra of the annealed graphene-on-Cu foil in Fig. 2(f) show that there is no change in the $G$ band frequency after annealing, indicating that there is no significant strain buildup during the heat treatment.

Since compressive strain can only occur during cooling, the heights of the macrosteps must depend sensitively on the cooling rate if indeed the macrosteps are formed during sample cooling. We compared samples prepared by both slow cooling (with a cooling rate of $\sim 4^{\circ} \mathrm{C} / \mathrm{min}$ ) and fast cooling (with a cooling rate of $\sim 100^{\circ} \mathrm{C} / \mathrm{min}$ ), and as shown in Figs. 2(d) and 2(e), no obvious difference in step heights is observed.

The above observations unambiguously show that SB can occur even without compressive strain and, therefore, it is unlikely that the key driving force for SB is the relaxation of compressive strain.

In the following, we consider an atomic description of the process of SB and explore the two essential conditions that must be satisfied for SB on the metal surface underlying a graphene layer-(i) the fast migration of metal adatoms beneath graphene and (ii) a driving force that lowers the formation energy of the whole system during SB.

Figures 3(a), 3(b) show the formation and diffusion of a $\mathrm{Cu}$ adatom on a $\mathrm{Cu}(111)$ surface with and without a graphene layer on the top (see computational details in the Supplemental Material [34]). The calculated formation
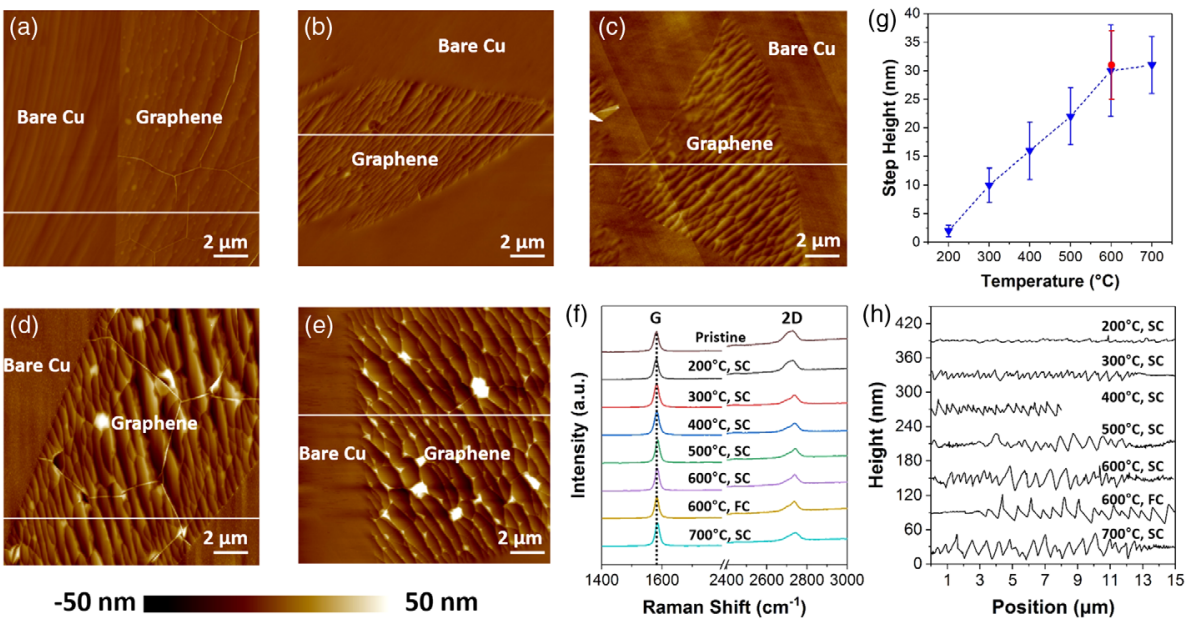

FIG. 2. (a)-(e) Atomic force microscope (AFM) topographic images of transferred graphene on single crystal Cu(111) foils after thermal annealing at $200{ }^{\circ} \mathrm{C}(\mathrm{a}), 300^{\circ} \mathrm{C}$ (b), $500{ }^{\circ} \mathrm{C}$ (c), and $600^{\circ} \mathrm{C}$ (d), (e) followed by slow cooling (a)-(d) and fast cooling (e), while images of 400 and $700^{\circ} \mathrm{C}$ are shown in Fig. S2 in the Supplemental Material [34]. (f) Raman spectra of the pristine and annealed graphene samples on single crystal $\mathrm{Cu}(111)$ foils. (g) Plot of step heights versus the annealing temperatures under both slow cooling (blue) and fast cooling (red) conditions. (h) AFM height profiles of the samples shown in (a)-(e) and in Fig. S2 [34]. In (f) and (h), SC and FC represent slow cooling and fast cooling, respectively. 

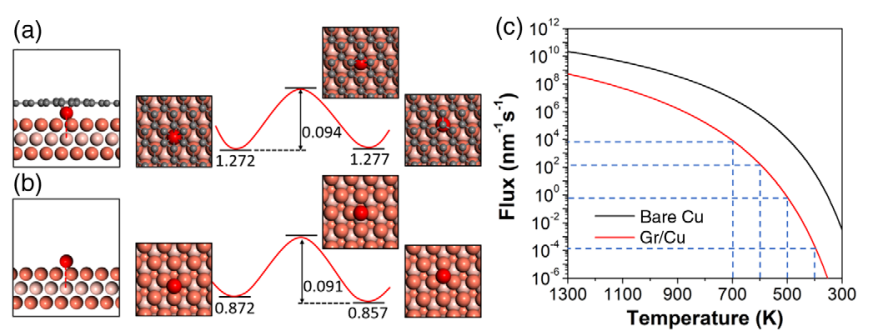

FIG. 3. (a),(b) Side views of a $\mathrm{Cu}$ atom on a $\mathrm{Cu}(111)$ surface with and without a graphene overlayer, top views of atom diffusion, and schematic plots showing formation energies and diffusion barriers in eV. Gray, red, and orange balls represent $\mathrm{C}$ atoms, $\mathrm{Cu}$ adatom, and $\mathrm{Cu}$ atoms in the substrate, respectively. (c) Flux of $\mathrm{Cu}$ atoms on bare $\mathrm{Cu}$ and graphene-covered $\mathrm{Cu}$ surfaces as a function of temperature.

energies of the adatom in the two cases are, respectively, 1.272 and $0.857 \mathrm{eV}$. The concentration of adatoms can be estimated according to the equation $\rho=e^{-E_{f} / k T}$, where $E_{f}$ is the formation energy, $k$ is the Boltzmann constant, and $T$ is the temperature. The calculated barriers to the diffusion of a $\mathrm{Cu}$ adatom with and without a graphene overlayer are both very low, namely, 0.094 and $0.091 \mathrm{eV}$, respectively. Based on these values, the frequency of hopping of an adatom on a $\mathrm{Cu}(111)$ surface can be roughly estimated from the relation $f=(k T / h) \times e^{-E_{b} / k T}$, where $E_{b}$ is the diffusion barrier and $h$ is the Planck constant. The flux of adatoms on a metal surface, defined as the number of atoms passing a distance of unit length on the metal surface in unit time can be estimated as

$$
F=(1 / a) \times \rho \times f=(k T / a h) \times e^{-\left(E_{f}+E_{b}\right) / k T},
$$

where $a=0.256 \mathrm{~nm}$ is the lattice constant of $\mathrm{Cu}$. The values for the flux of $\mathrm{Cu}$ atoms on bare $\mathrm{Cu}$ and graphene-covered $\mathrm{Cu}$ surfaces as a function of temperature are shown in Fig. 3(c), from which we can see that at the temperature of graphene CVD growth, there is sufficient flux of $\mathrm{Cu}$ atoms to initiate $\mathrm{SB}$ on the graphene-covered $\mathrm{Cu}(111)$ surface (i.e., there are $\sim 10^{9}$ atoms passing a distance of $1 \mathrm{~nm}$ length on the metal surface in $1 \mathrm{~s}$ at $T \sim 1300 \mathrm{~K}$ ), as long as there is a driving force to stabilize the bunched steps. At the temperatures of $700,600,500$, and $400 \mathrm{~K}$, the flux is greatly reduced to $10^{4}, 10^{2}, 1$, and $10^{-4}$ atoms $\mathrm{nm}^{-1} \mathrm{~s}^{-1}$, respectively. To form a macrostep of $10 \mathrm{~nm}$ high (or $\sim 50$ atoms thick) with an adjacent distance of $200 \mathrm{~nm}$, the relocation of $\sim 2 \times 10^{4}$ atoms during a reasonable, experimentally feasible time period (e.g., $10 \mathrm{~min}$ ) is required [34]. Thus, we estimate that the minimum flux required to form an observable metal step on the $\mathrm{Cu}$ surface is roughly $\sim 30$ atoms nm $\mathrm{nm}^{-1} \mathrm{~s}^{-1}$. From Fig. 3(c), we find that the minimum temperature required to have such a flux is $\sim 570 \mathrm{~K}$ (or $300^{\circ} \mathrm{C}$ ). This estimation is in excellent agreement with the experimental observation shown in Fig. 2, where we observe that SB becomes apparent for those foils annealed at $T>300^{\circ} \mathrm{C}$.
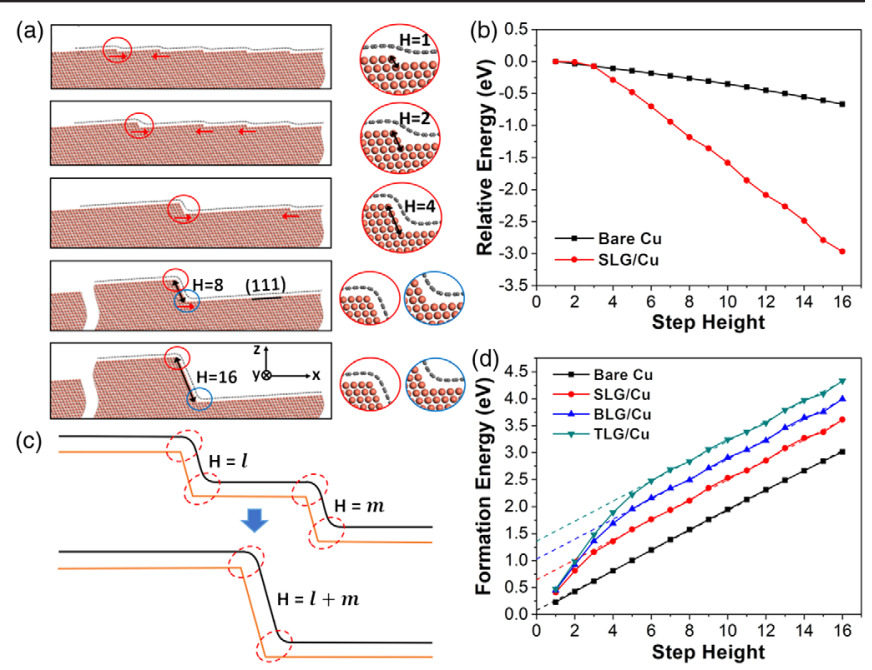

FIG. 4. (a) The process of the $\mathrm{Cu}(111)$ surface SB beneath a graphene layer. A series of images from top to bottom showing the increase in height of the highest step in each configuration, marked by black arrows with $H=1,2,4,8$, and 16. (b) The relative energy variation of bare $\mathrm{Cu}$ (black line) and SLG-covered $\mathrm{Cu}$ (red line) during the SB process (data for BLG and TLGcovered $\mathrm{Cu}$ are shown in Fig. S6 in the Supplemental Material [34]). (c) Schematic illustrations showing the different ways of bending of the graphene overlayer during the SB process. (d) Formation energies of bare $\mathrm{Cu}$ (black line) and $\mathrm{Cu}$ covered by SLG (red line), BLG (blue line), and TLG (green line), as a function of the step height.

In order to determine the nature of the driving force for SB beneath the graphene overlayer, we consider the process of SB of 16 single atomic steps covered with a graphene layer (Fig. S5 in the Supplemental Material [34]). Figure 4(a) shows the atomic process taking place during the process of SB on a $\mathrm{Cu}$ surface beneath a single finite graphene layer (like an island), during which, 16 single metal steps gradually bunch together to form a 16-times larger metal step eventually. The relative variation of energy during the SB process with and without the presence of a graphene overlayer are shown in Fig. 4(b). It is clearly seen that the total energy of the system reduces by $\sim 3 \mathrm{eV}$ per unit cell after $\mathrm{SB}$ in the presence of a graphene overlayer. This value is significantly larger than that for the bare $\mathrm{Cu}$ surface, which is only $\sim 0.5 \mathrm{eV}$ per unit cell. The above calculation clearly shows that the presence of graphene facilitates SB of the underlying $\mathrm{Cu}$ entirely in the absence of compressive strain relaxation.

The illustration in Fig. 4(c) demonstrates the origin of the driving force of metal surface SB beneath a graphene layer, where we see that graphene on a metal surface with two small steps is bent 4 times in order to tightly adhere to the stepped surface, whereas, it only bends twice if the two steps are bunched together to form a single large step. Thus, the curvature energy of the overlying graphene is reduced after SB because of relaxation of bending strain, which provides the dominant driving force for SB to occur on the underlying metal surface. 


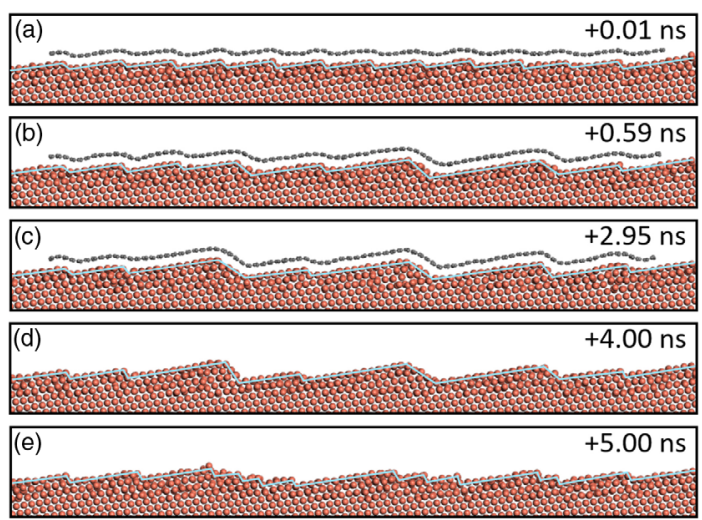

FIG. 5. Cu surface SB beneath the adlayer graphene (a)-(c) and the dissociation of the bunched steps (d),(e) after graphene removal observed in a MD simulation.

Figure 4(d) shows the formation energies of metal macro steps on a bare metal surface, along with those for single, bi-, and tri- layer graphene (SLG, BLG, and TLG)-covered metal surfaces as a function of the step height [34]. A linear relationship between the formation energy and the step height is obtained for all the different types of metal surfaces. For macrosteps that are sufficiently large, the formation energy of a graphene-covered step is higher than that on the bare metal surface. This energy difference is a constant value and is due to the higher bending energy of the overlying graphene film; the thicker the covered graphene layer, the larger is the difference in formation energy. The forces driving SB, defined as the decrease in energy when two steps are bunched together to form a larger step, are determined from the intercepts of the dashed lines with the $y$ axis [Fig. 4(d)] and the values are 0.07, $0.65,1.03$, and $1.36 \mathrm{eV}$ per unit cell (or 0.27, 2.54, 4.03, and $5.32 \mathrm{eV} / \mathrm{nm}$ ), respectively, for the four systems, namely, bare $\mathrm{Cu}, \mathrm{SLG} / \mathrm{Cu}, \mathrm{BLG} / \mathrm{Cu}$, and TLG/Cu.

To further approve the above analysis, molecular dynamics (MD) simulations (see method and movie in Supplemental Material [34]) were implemented to explore the process of SB beneath the graphene adlayer and one typical simulation trajectory is shown in Fig. 5. It can be clearly seen that the 12 single atomic steps appeared in the initial configuration [Fig. 5(a)] gradually bunch together and eventually two threefold steps and one twofold step are clearly seen after $\sim 3$ ns MD simulation [Figs. 5(b), 5(c)]. These large steps beneath the graphene adlayer are highly stable and their dissociation was never seen. While, after the graphene adlayer was removed [Fig. 5(d)], all the bunched steps are quickly dissociated and 12 single atomic steps appear on the metal surface again [Fig. 5(e)]. The MD simulation clearly shows the critical role of graphene coverage in metal surface SB and ambiguously validates the new mechanism of the graphene coverage induced metal surface SB.

The above calculation and analysis also show that the driving force for metal surface SB covered with a BLG or a
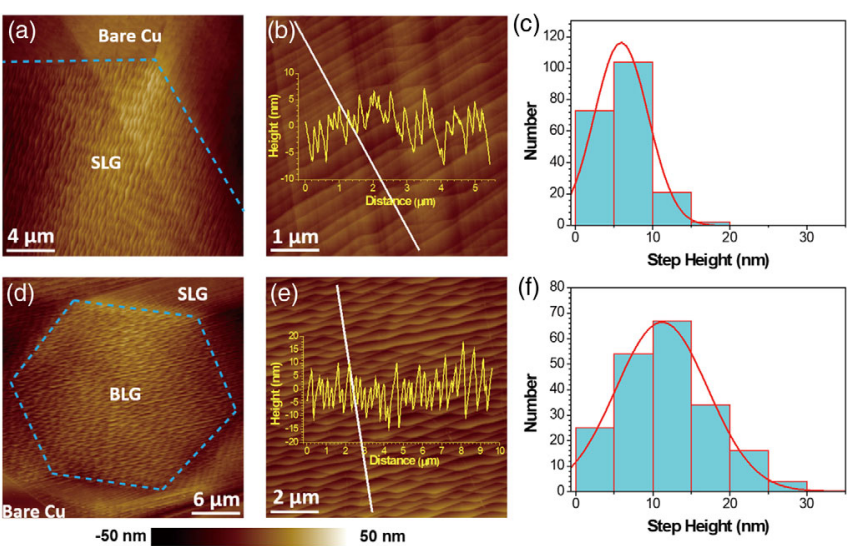

FIG. 6. AFM images and step height distributions of $\mathrm{Cu}(111)$ surfaces covered with SLG and BLG. AFM images, height profiles, and statistics of step height distributions of (a)-(c) SLG and (d)-(f) BLG.

TLG should be larger than that with a SLG as overlayer. To test this prediction, we carried out further experiments to measure the heights of metal steps beneath SLG- or BLGcovered $\mathrm{Cu}(111)$ surfaces and the results are shown in Fig. 6 [34]. For a SLG-covered $\mathrm{Cu}(111)$ surface, the step heights range from 3-10 $\mathrm{nm}$, whereas, the step heights under a BLG range from 5-20 nm. This result further validates the origin of the driving force for $\mathrm{SB}$ and also the theoretical prediction that $\mathrm{SB}$ of the metal substrate is more prominent when covered by a thicker multilayer graphene as compared to when covered by SLG.

Based on the new insights gained on the mechanism of $\mathrm{SB}$, we note that $\mathrm{SB}$ of a surface covered by a $2 \mathrm{D}$ material should be a general phenomenon, if the fast diffusion of the adatoms is activated at the interface. Such behavior has been observed in CVD grown $h$-BN [52,54], and during the growth of graphene on $\mathrm{Pt}$ and $\mathrm{Ni}$ surfaces $[7,8,55,56]$. Hence, the SB of the substrate should be taken into consideration during the processing of $2 \mathrm{D}$ materials for various applications.

In summary, we have carried out a detailed analysis of step bunching in graphene-covered metal surfaces. Our results unambiguously show that $\mathrm{SB}$ is not driven by relaxation of compressive strain in graphene but is enabled by the fast diffusion of metal adatoms beneath the graphene layer and driven by the release of local bending energy of the graphene overlayer in the vicinity of surface steps on the metal. Our analysis explains a number of experimental observations such as the dependence of SB on temperature, number of graphene layers, and increased $\mathrm{SB}$ at the interface between 2D and substrate materials.

The authors acknowledge support from the Institute for Basic Science (IBS-R019-D1) of South Korea.

D. Y., D. L., and Z.-J.W. contributed equally to this work. 
*willinger@fhi-berlin.mpg.de

†ruofflab@gmail.com

\#.ding@unist.ac.kr

[1] X. Li et al., Science 324, 1312 (2009).

[2] Y. Hao et al., Science 342, 720 (2013).

[3] X. Xu et al., Nat. Nanotechnol. 11, 930 (2016).

[4] A. Reina, X. Jia, J. Ho, D. Nezich, H. Son, V. Bulovic, M. S. Dresselhaus, and J. Kong, Nano Lett. 9, 30 (2009).

[5] K. S. Kim, Y. Zhao, H. Jang, S. Y. Lee, J. M. Kim, K. S. Kim, J.-H. Ahn, P. Kim, J.-Y. Choi, and B. H. Hong, Nature (London) 457, 706 (2009).

[6] T. Gao, S. Xie, Y. Gao, M. Liu, Y. Chen, Y. Zhang, and Z. Liu, ACS Nano 5, 9194 (2011).

[7] L. Gao et al., Nat. Commun. 3, 699 (2012).

[8] Z.-J. Wang, J. Dong, Y. Cui, G. Eres, O. Timpe, Q. Fu, F. Ding, R. Schloegl, and M.-G. Willinger, Nat. Commun. 7, 13256 (2016).

[9] P. W. Sutter, J.-I. Flege, and E. A. Sutter, Nat. Mater. 7, 406 (2008).

[10] Y. Pan, H. Zhang, D. Shi, J. Sun, S. Du, F. Liu, and H. Gao, Adv. Mater. 21, 2777 (2009).

[11] S. Roth, J. Osterwalder, and T. Greber, Surf. Sci. 605, L17 (2011).

[12] M. Sicot, S. Bouvron, O. Zander, U. Rüdiger, Y. S. Dedkov, and M. Fonin, Appl. Phys. Lett. 96, 093115 (2010).

[13] N. Blanc, F. Jean, A. V. Krasheninnikov, G. Renaud, and J. Coraux. Phys. Rev. Lett. 111, 085501 (2013).

[14] C. Herbig, T. Knispel, S. Simon, U. Schröder, A. J. Martínez-Galera, M. A. Arman, C. Teichert, J. Knudsen, A. V. Krasheninnikov, and T. Michely, Nano Lett. 17, 3105 (2017).

[15] Z. Yan, J. Lin, Z. Peng, Z. Sun, Y. Zhu, L. Li, C. Xiang, E. L. Samuel, C. Kittrell, and J. M. Tour, ACS Nano 6, 9110 (2012).

[16] A. Mohsin et al., ACS Nano 7, 8924 (2013).

[17] L. Gan and Z. Luo, ACS Nano 7, 9480 (2013).

[18] V. L. Nguyen et al., Adv. Mater. 27, 1376 (2015).

[19] T. Wu, G. Ding, H. Shen, H. Wang, L. Sun, D. Jiang, X. Xie, and M. Jiang, Adv. Funct. Mater. 23, 198 (2013).

[20] C. Wang et al., Sci. Rep. 4, 4537 (2014).

[21] H. Zhou, W. J. Yu, L. Liu, R. Cheng, Y. Chen, X. Huang, Y. Liu, Y. Wang, Y. Huang, and X. Duan, Nat. Commun. 4, 2096 (2013)

[22] S. Bae et al., Nat. Nanotechnol. 5, 574 (2010).

[23] X. Xu et al., Science bulletin 62, 1074 (2017).

[24] T. Wu et al., Nat. Mater. 15, 43 (2016).

[25] G. H. Han, F. Güneş, J. J. Bae, E. S. Kim, S. J. Chae, H.-J Shin, J.-Y. Choi, D. Pribat, and Y. H. Lee, Nano Lett. 11, 4144 (2011).

[26] Y. Zhang, T. Gao, Y. Gao, S. Xie, Q. Ji, K. Yan, H. Peng, and Z. Liu, ACS Nano 5, 4014 (2011).

[27] Y. Zhang et al., Carbon 96, 237 (2016).

[28] G.-X. Ni et al., ACS Nano 6, 1158 (2012).

[29] K. Hayashi, S. Sato, and N. Yokoyama, Nanotechnology 24, 025603 (2013).
[30] X. H. Kong, H. X. Ji, R. D. Piner, H. F. Li, C. W. Magnuson, C. Tan, A. Ismach, H. Chou, and R. S. Ruoff, Appl. Phys. Lett. 103, 043119 (2013).

[31] J. H. Kang, J. Moon, D. J. Kim, Y. Kim, I. Jo, C. Jeon, J. Lee, and B. H. Hong, Nano Lett. 16, 5993 (2016).

[32] J.-S. Yu, X. Jin, J. Park, D. H. Kim, D.-H. Ha, D.-H. Chae, W.-S. Kim, C. Hwang, and J.-H. Kim, Carbon 76, 113 (2014).

[33] D. Luo et al., Chem. Mater. 29, 4546 (2017).

[34] See Supplemental Material at http://link.aps.org/ supplemental/10.1103/PhysRevLett.120.246101 for more experimental and theoretical details, and Refs. [35-44].

[35] K. S. Novoselov, A. K. Geim, S. V. Morozov, D. Jiang, Y. Zhang, S. V. Dubonos, I. V. Grigorieva, and A. A. Firsov, Science 306, 666 (2004).

[36] G. Kresse and J. Furthmüller, Comput. Mater. Sci. 6, 15 (1996).

[37] G. Kresse and J. Furthmüller, Phys. Rev. B 54, 11169 (1996).

[38] J. P. Perdew, K. Burke, and M. Ernzerhof, Phys. Rev. Lett. 77, 3865 (1996).

[39] P. E. Blöchl Phys. Rev. B 50, 17953 (1994).

[40] S. Grimme J. Comput. Chem. 27, 1787 (2006).

[41] G. Henkelman, B. P. Uberuaga, and H. Jónsson, J. Chem. Phys. 113, 9901 (2000).

[42] S. Plimpton J. Comput. Phys. 117, 1 (1995).

[43] M. I. Mendelev, M. J. Kramer, C. A. Becker, and M. Asta, Philos. Mag. 88, 1723 (2008).

[44] S. J. Stuart, A. B. Tutein, and J. A. Harrison, J. Chem. Phys. 112, 6472 (2000).

[45] C. J. Smithells, in Smithells Metals Reference Book, edited by W. F. Gale and T. C. Tetemeier (Oxford, Elsevier, Butterworth-Heinemann, 2004), p. 14.

[46] M. Pozzo, D. Alfè, P. Lacovig, P. Hofmann, S. Lizzit, and A. Baraldi, Phys. Rev. Lett. 106, 135501 (2011).

[47] D. Yoon, Y.-W. Son, and H. Cheong, Nano Lett. 11, 3227 (2011).

[48] S. J. Chae et al., Adv. Mater. 21, 2328 (2009).

[49] H. Lim, J. Jung, R. S. Ruoff, and Y. Kim, Nat. Commun. 6, 8601 (2015).

[50] J. Tian, H. Cao, W. Wu, Q. Yu, N. P. Guisinger, and Y. P. Chen, Nano Lett. 12, 3893 (2012).

[51] D. W. Kim, J. Lee, S. J. Kim, S. Jeon, and H.-T. Jung, J. Mater. Chem. C 1, 7819 (2013).

[52] T. Gao, X. Song, H. Du, Y. Nie, Y. Chen, Q. Ji, J. Sun, Y. Yang, Y. Zhang, and Z. Liu, Nat. Commun. 6, 6835 (2015).

[53] J. Zabel, R. R. Nair, A. Ott, T. Georgiou, A. K. Geim, K. S. Novoselov, and C. Casiraghi, Nano Lett. 12, 617 (2012).

[54] G. E. Wood, A. J. Marsden, J. J. Mudd, M. Walker, M. Asensio, J. Avila, K. Chen, G. R. Bell, and N. R. Wilson, 2D Mater. 2, 025003 (2015).

[55] D. Usachov, A. M. Dobrotvorskii, A. Varykhalov, O. Rader, W. Gudat, A. M. Shikin, and V. K. Adamchuk, Phys. Rev. B 78, 085403 (2008).

[56] T. Iwasaki, H. J. Park, M. Konuma, D. S. Lee, J. H. Smet, and U. Starke, Nano Lett. 11, 79 (2011). 